\title{
BMJ Open DetectaWeb Project: study protocol of a web-based detection of mental health of children and adolescents
}

\author{
Jose A Piqueras, ${ }^{1}$ Mariola Garcia-Olcina, ${ }^{1}$ Maria Rivera-Riquelme, ${ }^{1}$ \\ Tiscar Rodriguez-Jimenez, ${ }^{2}$ Agustin E Martinez-Gonzalez, ${ }^{3}$ Pim Cuijpers ${ }^{4,5}$
}

To cite: Piqueras JA, GarciaOlcina M, Rivera-Riquelme M, et al. DetectaWeb Project: study protocol of a webbased detection of mental health of children and adolescents. BMJ Open 2017;7:e017218. doi:10.1136/ bmjopen-2017-017218

- Prepublication history for this paper is available online. To view these files please visit the journal online (http://dx.doi. org/10.1136/bmjopen-2017017218).

Received 8 April 2017

Revised 5 August 2017

Accepted 10 August 2017

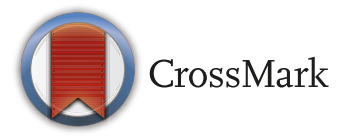

${ }^{1}$ Department of Health Psychology, Miguel Hernandez University of Elche, Elche, Spain ${ }^{2}$ Department of Psychology, Catholic University of Murcia,

Murcia, Spain

${ }^{3}$ Department of Developmental Psychology and Didactics, University of Alicante, Alicante, Spain

${ }^{4}$ Department of Clinical Psychology, Vrije Universiteit Amsterdam, Amsterdam, The Netherlands

${ }^{5}$ The Netherlands \& EMGO Institute for Health and Care Research, Amsterdam, The Netherlands

Correspondence to Dr Jose A Piqueras; jpiqueras@umh.es

\begin{abstract}
Introduction Traditionally, research and practice addressing mental health has been conducted within a unidimensional model, explaining mental health as the presence/absence of psychological problems (distress or psychopathological symptoms). More recently, accumulative evidence has supported a bi-dimensional model, which conceptualises psychological problems and psychological strengths (well-being) as related-yetdistinct continua that, when considered together, yields a more comprehensive understanding of individuals. The DetectaWeb Project is a web-based early detection assessment of the mental health continuum (MHC) for children and adolescents and aims to: develop a webbased platform for screening of the MHC, including both psychological problems (anxiety, depression and suicidality) and personal strengths (emotional, cognitive/ psychological and social aspects); examine the prevalence of the MHC among children and adolescents; and analyse key determinants (risk and protective factors) of the MHC. Methods and analysis We will conduct an ongoing, multicentre observational, cross-sectional study of Spanish children and adolescents. Participants will be assessed through a web-based platform. In order to validate the web-based screening system, a clinical reappraisal will be completed among a subsample of respondents. To elucidate youth's levels of subjective distress and wellbeing, we will include measures of anxiety, depression, suicidality, well-being, externalising problems and sociodemographic variables.

Ethics and dissemination Ethical approval has been obtained at all sites. All participants will sign a free and informed consent form before the assessment. Results of the study will be communicated during national and international meetings in the field of clinical and health psychology with children and adolescents. Publications will be sought in journals usually read by psychologists or psychiatrists involved in the development of epidemiological studies and interventions for mental health promotion based on resilience building for children and adolescents.
\end{abstract}

\section{INTRODUCTION}

The World Health Organization (WHO) has stated that there is no health without mental health. Mental health is 'a complete state of physical, mental, and social well-being, and

\section{Strengths and limitations of this study}

The DetectaWeb Project is an ongoing, multicentre, observational, cross-sectional and web-based study of the mental health continuum of Spanish children and adolescents, both from schools and mental health units.

- No interference with the educational or clinical decision-making process of school counsellors or physicians, respectively.

- Collected information equals the knowledge of mental health status and the specific needs for care.

- Representativeness of the sample may limit the external validity of the study.

not only the absence of diseases or illnesses'. Therefore, mental health is much more than the absence of mental disorders. ${ }^{1}$ Consequently, different international organisations, such as the European Commission or the WHO, have committed to promoting mental health and preventing mental disorders in children and adolescents. For example, the WHO in its World Assembly of Health in 2013 approved a plan to promote mental health from 2013 to $2020 .^{2}$

Traditionally, research and practice addressing social and emotional health has been conducted within a unidimensional model of mental health. This model assumes that psychological distress is the opposite of psychological well-being, indicating that a reduction in distress is automatically accompanied, or even is synonymous, with an increase in well-being. ${ }^{3-5}$ In other words, conventional research on mental health has almost exclusively focused on models that explain psychopathology as psychological problems or distress, neglecting other aspects such as personal strengths or well-being, which offers a more integral vision of mental health. ${ }^{6}$ Some examples of how traditional research and practice have assessed mental health from this framework are surveillance 
surveys that focused on risk behaviours; symptom tools to identify clinical significant problems related to specific disorders such as anxiety or depression; or multi- or single-informant referrals of students experiencing mental health problems who rely on teachers, parents, etc. Furthermore, focusing exclusively on problems is limited as this approach is only applicable to $15 \%-20 \%$ of the population (ie, those presenting symptoms) and over-identifying externalising symptoms does not provide any information on positive potentials or strengths. ${ }^{7}$

On the other hand, over the past two decades, different lines of research findings have challenged this unidimensional model, providing evidence for a bi-dimensional model of mental health, which conceptualises distress and well-being as related yet continuously distinct concepts, that, when considered together, offers a more complete and richer understanding of the human condition. ${ }^{4}$ The number of authors who consider mental health as a continuum is increasing. This new approach has a series of advantages: it follows a dual factor model of mental health; it is applicable to $100 \%$ of individuals; and it fosters strong, successful people instead of just preventing psychological problems. ${ }^{7}$ Moreover, to measure mental health, both the presence of psychopathology or emotional distress and subjective well-being must be considered. ${ }^{3} 7$ A more representative model of this new theoretical approach is provided by Ryff \& Keyes, ${ }^{6}$ which has several names: two-dimensional model of mental health, MHC, complete mental health status, well-being and mental health, or dual mental health. This new scientific approach originally examined adult samples in the past 10 to 20 years, but little research has been conducted with children and adolescents.

Concerning children and adolescents, recent studies have supported this bi-dimensional model of mental health, ${ }^{8-11}$ reporting that children who show high levels of psychological distress also show low levels of subjective well-being, or that both the presence of psychological distress and the absence of psychological well-being are associated with academic functioning, considering that both indicators provide a higher predictive value in adjustment to school. Consequently, some authors emphasise that the "bi-dimensional model of mental health suggests that efforts seeking to cultivate positive mental health warrant shared attention with efforts aiming to ameliorate psychological impairment and symptoms ${ }^{12}$ Consequently, in this article, we believe that any mental health assessment should measure two constructs: subjective distress, understood as present psychopathological symptoms, and subjective well-being, understood as psychological, emotional and social aspects of well-being. ${ }^{12}$

Different authors consider that subjective distress is determined largely by the presence of emotional or internalising symptoms: anxiety, depression ${ }^{13}{ }^{14}$ and suicidality. ${ }^{15}$ Accordingly, internalising disorders can be differentiated in two sets: distress disorders such as generalised anxiety disorder (GAD), post-traumatic stress disorder (PTSD), major depression (MD) and dysthymic disorder (DD), and fear/anxiety disorders (such as panic and phobias). ${ }^{16-18}$ From a broader point of view, according to relevant authors in this field, such as Kessler and colleagues, Clark and Watson, Krueger, and Watson, ${ }^{14}$ 16-18 psychological distress can be conceptualised as the presence of symptoms of some of the emotional disorders without differentiating between them: however, these studies did not include suicidality as a type of distress. Per a recent meta-analytic review, the global prevalence of anxiety and depressive disorders in youths is $6.50 \%$ (95\% CI: $4.70-9.10)$ and $2.60 \%$ (95\% CI: $1.70-3.90)$, respectively, ${ }^{19}$ with a marked comorbidity between both disorders. ${ }^{9} 10$ Several studies among the general population show that common mental disorders are related to suicidality $(\mathrm{ORs}=2.07-10.06) .{ }^{20}{ }^{21}$ Furthermore, among adolescents aged 10 to 24 years, suicide is the second leading cause of death. ${ }^{22}$

Regarding well-being, there are two philosophical traditions in its study: a eudaimonic perspective and a hedonic perspective (for a further description, $\operatorname{se}^{23}$ ). From a eudaimonic perspective, the most used concept is psychological well-being, corresponding to the development of one's true self, self-acceptance and personal growth among others. ${ }^{24}$ This includes the capacity for self-development, autonomy, self-acceptance and competence. ${ }^{25}$ Whereas, for hedonism, the preferred construct is subjective well-being, which is the more accepted concept at present and includes two components: life satisfaction (a cognitive dimension that is more stable and similar to a trait) and affective balance between positive and negative affect (an emotional dimension that is more immediate).$^{2627} \mathrm{~A}$ high subjective well-being corresponds with people experiencing high life satisfaction and positive affect, accompanied by low levels of negative affect. Other authors, who pursue to integrate both traditions, assert that subjective well-being is now largely accepted as covering two perspectives: positive psychological functioning, good relationships with othersand self-realisation (the eudaimonic perspective); and the subjective experience of happiness (affect) and life satisfaction (the hedonic perspective). The prevalence rates in adolescents aged 12 to 18 years is approximately $38 \%$ for high levels of positive mental health or flourishing and about $6 \%$ for low levels of positive mental health or languishing. ${ }^{28}$

All these data suggest the need for mental health services and professionals to pay attention to emotional status, understood as well-being and distress. However, it is very rare to find preventive programmes that foster wellbeing and positive emotions, and to find mental health professionals who foster well-being and positive emotions as healthy vital elements. ${ }^{23}$ In this sense, it is well known that the best setting to implement programmes that focus on the promotion, prevention and detection of mental health are schools. ${ }^{29}$ Some studies have used the Internet in school settings to support the screening, detection and identification of mental disorders, ${ }^{30-32}$ showing that the Internet facilitates the promotion, preventionand interventions of mental health in adolescents. ${ }^{33}$ 
In addition, the Internet offers other advantages for participants and researchers, such as the reduction of work burden, a greater disclosure for individuals and better access to hard-to-reach populations. ${ }^{34}$ However, despite the great rise of clinical research on the application of the Internet and new technologies, few studies have addressed the mental health continuum (MHC), which start from routine check-ups to the prevention of emotional distress ${ }^{35}$ and the promotion of psychological and emotional well-being, which can be used at schools or by family or paediatric physicians as a screening assessment prior to seeing a child psychologist/psychiatrist. Therefore, utilising the MHC would ensure all children who need it receive adequate psychological and psychiatric care. In the same line, there are some previous detection programmes for adolescents that function as routine check-ups: the TeenScreen National Centre of checks of mental health at the University of Columbia (http://mhaok.org/what-we-do/youth-outreach/ columbia-teenscreen/), the American Academy of Pediatrics (www.aap.org), and CoVitality, an online social-emotional health students survey (http://www. covitalityapp.com/).

Therefore, considering the goal of the WHO is to promote mental health, there has been an increase in the number of people with emotional disorders, and there is a shortage of online assessment programmes that prevent mental disorders and promote mental health in children and adolescents, we initiated the DetectaWeb Project. This web-based program promotes an early detection of the MHC, which evaluates both psychological distress or emotional symptoms (anxiety, depression and suicidality) (DetectaWeb-Distress) and subjective well-being matters relating to emotional, psychological and social needs such as good emotional balance (eg, positive vs. negative affect), life satisfaction and positive psychological functioning (eg, good relationships with others) (DetectaWeb-Well-being). Our study will be applied to children and adolescents aged 8 to 18 years in the hope of being used as a screening tool at children's first visit to mental health centres or during routine checkups at schools.

In summary, compared with traditional measures and programmes aimed at the early detection of mental disorders, very few studies have examined the utility of assessing the MHC in children and adolescents by measuring subjective distress and well-being.

\section{Objective}

This project aims to serve the following purposes: develop a web-based platform for early screening of the MHC, including both psychological problems (DetectaWeb-Distress: anxiety, depression and suicidality) and personal strengths (DetectaWeb-Well-being: emotional, cognitive/ psychological and social aspects); examine the prevalence of MHC problems among children and adolescents; and analyse key determinants (risk and protective factors) of the MHC.

\section{MATERIAL AND METHODS}

\section{Study design}

The DetectaWeb Project is an ongoing, multicenre, observational, cross-sectional study of children and adolescents from Spanish centres of primary and secondary education, as well as from Children and Adolescents Mental Health Units (CAMHU). Participants will be assessed once during the academic year. The surveys will be administered through an online, secure web-based platform. A clinical reappraisal will be completed among a subsample of respondents. Within the bi-dimensional model of mental health, an instrumental study of adaptation and validation of different instruments for assessing mental health (subjective distress and well-being), and a prevalence study of mental health (distress, well-being, flourishing and languishing) in children and adolescents will be conducted.

\section{Setting}

Data are being collected from primary, secondary and high-school centres and from CAMHU in Alicante (Community of Valencia, Spain) in four academic years. The study will be conducted in four phases: the development of the web platform and adaptation of MHC measures; a pilot study providing preliminary data of the DetectaWeb project; web-based screening and detection of MHC in school samples; and clinical reappraisal interviews addressing MHC in clinical samples.

Concerning the community-based sample, we will follow a convenience sampling method. Therefore, eight centres comprising primary and secondary education and high-school centres through a random cluster sampling of the main counties (North, Central and South) of the Province of Alicante (Community of Valencia, Spain) will participate. To warrant that all geographic areas of the province were represented, four public and four semi-private schools were randomly selected. For this, the primary sampling units were the geographical counties of the province of Alicante, whereas the secondary units were the schools in each region (proportional randomly selected) and, finally, tertiary units were classrooms.

Regarding the clinical sample, we will also follow random cluster sampling. Therefore, we will select CAMHU from the three main counties of Alicante (North, Central and South). The geographical areas will comprise the primary sampling units, CAMHU will comprise the secondary units ('Unidades de Salud Mental Infanto-Juvenil') and mental health professionals from CAMHU will comprise the tertiary units.

\section{Participants}

\section{School-based sample}

Inclusion criteria: aged 8-18 years and enrolled in grades 3-6 of elementary education, 1-4 of secondary education or 1-2 of higher education.

Exclusion criteria: poor knowledge of the Spanish language; parents or guardians do not provide informed consent or children older than 2 years do not provide 
informed consent (compulsory according Spanish law); and students do not attend class the day of the assessment.

Based on eligibility criteria, we estimate than about 2000 students will be eligible to participate.

\section{Clinical sample}

Inclusion criteria: service users aged between 8 to 18 years in their first or second visit to the CAMHU; whose reason for consultation is anxiety/depression symptoms $(n=75)$ or other symptoms $(n=75)$; and parents or tutors have to sign the authorisation to participate in the study, and children older than 12 years.

Exclusion criteria: refusing to participate in the study and patients immersed in psychological or psychiatric therapy.

Based on eligibility criteria, we estimate that about 150 patients will be eligible to participate.

\section{Data collection online platform (registration and verification)}

To participate in this study, eligible participants are invited to complete the study survey in their own classrooms providing them with some of our research team's tablets with a Wi-Fi connection or in their own computer laboratories at each school. Concerning CAMHU, a specific space will be enabled to complete the survey by means of our tablets or their own computers or devices. To ensure the enforcement of data protection and security of clinical data information, respondents will be assigned a code that only they or the counsellor/school director knows, making the survey anonymous. In addition, before completing the survey, they will be informed about where their data will be collected and the purpose of this study. Likewise, the instructions will be loudly communicated collectively in the second and third phases and individually in the fourth phase by a research assistant who always accompanies participants.

\section{Timing of assessment}

The study will be conducted for four academic years between 2016-2020 (September to June in Spain). We will use a school year for each phase of our study. Respondents will complete the online survey in approximately $50 \mathrm{~min}$ and diagnostic interviews will take approximately 1.5 hours.

\section{Description and timing of the stages}

First stage: 'The development of the web platform and adaptation of MHC measures'

Children from at least two school centres (public and semi-private) will be selected. This point will be divided into two stages. The first stage (1.1 figure 1$)$ will be the creation of a web platform to collect the data (www.detectaweb.com/blog). The assessments will be conducted by using a LimeSurvey platform (www.limesurvey.org). LimeSurvey is an online platform for the creation and implementation of online surveys, with Likert-type responses and allowing for collected data that can be transformed for analysis with SPSS statistical software (version 24.0, SPSS, Inc., Chicago, IL). It will follow the international guidelines on computer-based and internet delivered testing. ${ }^{36} 37$

The second step (1.2 figure 1) will be the creation or adaptation of instruments that will allow us to assess subjective distress, the DetectaWeb-Distress scale and the validation of tools for the measurement of subjective wellbeing, the DetectaWeb-Well-being scale.

With respect to DetectaWeb-Distress, we will create a questionnaire for children and adolescents to identify distress and well-being (the brief Web-based Screening Questionnaire for the MHC - Distress), based on the Web-Based Screening Questionnaire for Common Mental Disorders (WSQ), consisting of 15 items. ${ }^{32}{ }^{36}$ For this, we will follow the international guidelines for the adaptation and creation of tests. ${ }^{37-39}$ First, we will translate and adapt the original WSQ into Spanish, following the standard forward-back-forward translation method. Second, we will install an expert panel of individuals who specialise in child and adolescent psychology, who will compare the translated version against the original WSQ

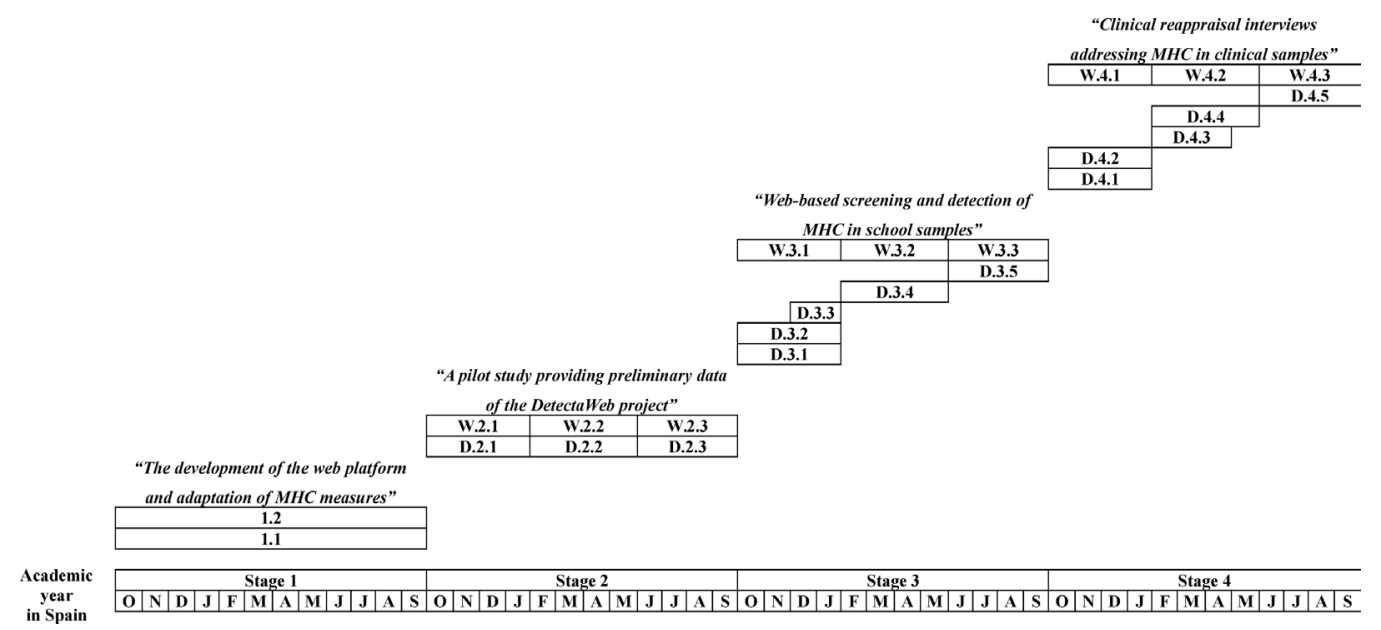

Figure 1 Timeline study design. The DetectaWeb Project Note. D, DetectaWeb Distress; W, DetectaWeb Well-being. Letters on the $\mathrm{X}$-axis denote months of the year in order. 
for comprehension, wording and response format. Third, these same experts will provide a bank of items to be able to more conveniently assess anxiety, depression and suicidality in children and adolescents. Fourth, a pilot study will be conducted and pertinent analyses will be performed to select the items with the best psychometric properties. At the same time, we will ensure all self-reported questionnaires measuring the MHC are translated into Spanish.

Concerning DetectaWeb-Well-Being, we will adapt, into an online format, the Mental Health Inventory (MHI-38 and MHI-5) and the Spanish version of the 5-item WHO Well-Being Index (WHO-5). We will also include the Stirling Children's Well-being Scale, ${ }^{40}$ the Social Emotional Health Survey-Secondary ${ }^{41}$ and the SEHS-Primary, the Trait Emotional Intelligence Questionnaire-Children Short Form (TEIQ-CSF) ${ }^{42}$ and the new Questionnaire for Children's Positive Emotions. ${ }^{43}$

\section{Second stage: 'A pilot study providing preliminary data of the DetectaWeb Project'}

A pilot study will be conducted with the assessment protocol. Regarding the assessment of distress, the objective of this phase of the study will be to analyse the preliminary psychometric properties of the DetectaWeb-Distress scale. This phase will be divided into three parts: online assessment of distress (anxiety, depression and suicidality) (see D.2.1 figure 1); data analysis (see D.2.2. figure 1); and dissemination and publication of the results (see D.2.3 figure 1).

Regarding well-being assessment, it will be divided into the same phases as the first part (see from W.2.1 to W.2.3. in figure 1).

\section{Third stage: 'Study of web-based screening and detection of MHC} in school samples'

The sample will consist of about 2000 schoolchildren from eight participating centres, with the inclusion and exclusion criteria indicated above. Regarding the assessment of distress, the study will be divided into five parts: web-based assessment of emotional distress (anxiety, depression and suicidality) (see D.3.1 figure 1); to promote the diagnostic accuracy of DetectaWeb, we will administer structured diagnostic clinical interviews to $20 \%$ of the sample with positive identification of high distress and low wellbeing (see D.3.2 figure 1); data analyses to obtain the psychometric properties of the DetectaWeb-Distress scale (see D.3.3 figure 1); based on the group and individual scores, reports will be delivered for participants (see D.3.4 figure 1); and dissemination and publication of results (see D.3.5 figure 1).

We will distinguish three types of reports: participants with low levels of distress; participants who present symptoms of distress; and children and adolescents who present distress symptoms compatible with a high probability of an emotional disorder (this last report will be addressed to the corresponding paediatrician or mental health professional).
Regarding DetectaWeb-Well-being, the study will be divided into three parts: web-based assessment of wellbeing. Concretely, we will assess good emotional balance, life satisfaction and positive psychological functioning such as good relationships with others (see W.3.1 figure 1); (data analyses to provide psychometric properties of used questionnaires (see W.3.2 figure 1); and (dissemination of results (see W.3.3 figure 1).

\section{Fourth stage: 'Clinical reappraisal interviews addressing MHC in} clinical samples'

Concerning the assessment of distress, we will perform five tasks: web-based psychological assessment of emotional distress (D.4.1 figure 1); structured diagnostic interviews with participants (D.4.2 figure 1); analyses of inter-raters' reliability (by means of the diagnosis assigned by two trained raters who will observe the recorded interviews). We will also have the clinical diagnoses 'as usual' by USMI's mental health professionals (D.4.3. figure 1); data analyses to obtain psychometric properties of instruments for the clinical sample (D.4.4 figure 1); and dissemination and publication of results (D.4.5 figure 1).

The assessment of well-being will be divided into three parts: web-based assessment of well-being in clinical populations (W.4.1, figure 1); data analysis to obtain psychometric properties of instruments (W.4.2 figure 1); and dissemination of results (W.4.3 figure 1).

\section{Data collection platform}

We will follow international recommendations and guidelines for computerised assessment ${ }^{38}{ }^{39}$ in the design and development of the data collection platform (DCP). The DCP is specially designed for the DetectaWeb Project, ensuring proper use, technical handling, quality control and security of the data.

\section{Study questionnaires}

Based on the bidimensional model of MHC, considering that mental health is not only the absence of mental disease but also the presence of well-being, our project gathered self-reported data about subjective distress and well-being. To obtain the level of distress and well-being among adolescents, we will include measures of anxiety, depression, suicidality, wellness, quality of life, emotional intelligence, gratitude and socio-demographic variables.

Per previous studies, ${ }^{14} 16-18$ we conceptualised subjective distress as the presence of symptoms of some of the main emotional disorders: $\mathrm{MD}$, majordepression; DD, persistentdepressive disorder or dysthymic disorder; GAD, generalised anxiety disorder; $\mathrm{SAD}$, separation anxiety disorder; SP, specific phobia; $\mathrm{SoPh}$, social phobia; $\mathrm{Pd} / \mathrm{Ag}$, panic disorder/agoraphobia; OCD, obsessive compulsive disorder; PTSD, posttraumatic stress disorder; and suicidal thoughts and behaviours (ie, ideation, plans and attempts). Both the use of paperand-pencil self-reported measures for young children's mental health, even with children with significant mental health problems, and the online-self-report measures with 
children as young as 8 years' old, have received increasing evidence. ${ }^{44}$ The questionnaire content is summarised in table 1 and is described in more detail below.

\section{Sociodemographic factors}

Age, sex, nationality and descriptive information about socio-economic status (SES) will be collected. Specifically, we will administer two SES measures:

- Family Affluence Scale (FAS) ${ }^{45}$ will be used to measure socioeconomic level. The FAS assesses household purchasing power or family wealth, and consists of four items about family car ownership, having an (own) unshared room, the number of computers at home and the number of times the child was on holidays in the past year. The FAS is scored in categories ranging from 0 to 7 : low (0-3), intermediate (4-5) and high (6-7). The scale was developed to reliably estimate family SES in (young) children using questions they are likely to know about. It has shown good criterion and construct validity in previous studies. ${ }^{46}$

- The Hollingshead scale ${ }^{47}$ was used to assess the social class distribution of parents. The SES index considers the education level and occupation of parents. In the case of a single-parent family, the SES index is calculated for the relative who lives with the child, and allows a classification of five levels: low, mid-low, medium, mid-high and high.

\section{Subjective distress}

Internalising and externalising disorder symptoms

- DetectaWeb-Distress Scale. This is a web-based screening questionnaire for emotional mental disorders, which was created in $2014 .{ }^{48}$ It consists of 30 items that assess anxiety disorders (SAD, SP, SoPh, GAD, Pd/Ag), PTSD, OCD, unipolar depressive disorders (MD and DD) and suicidality (ideation, plans and attempts). It follows a Likert-type response format $(0=$ never, $1=$ sometimes, $2=$ often and $3=$ always). A previous study has provided initial support for the reliability $(\alpha=0.87)$ and validity $(r=0.81)$ of the DetectaWeb-Distress Scale to assess anxiety, depression and suicidality in children and adolescents. ${ }^{48}$

- Revised Child Anxiety and Depression Scale, 30-item version $(R C A D S) .{ }^{49}$ The reduced 30-item RCADS ${ }^{49}$ is a self-report for evaluating anxiety and depression in children and adolescents. The scale comprises six subscales for evaluating symptoms of the following conditions: $\mathrm{Pd}$ / $\mathrm{Ag}$, SoPh, SAD, GAD, OCD and MD. The scale ranges from 0 to 3 (never, sometimes, often and always, respectively). The scale has shown excellent psychometric properties, equivalent to the full version, with Spanish populations $(\alpha=0.87){ }^{50}$

- The Strengths and Difficulties Questionnaire $(S D Q){ }^{51}$ The SDQ is widely used for the assessment of different emotional and behavioural problems in children and adolescents. The SDQ comprises 25 statements distributed across five subscales: emotional symptoms, conduct problems, hyperactivity, peer problems and prosocial behaviour. In this study, we will use the Spanish version of the self-reported SDQ for 11 to 17 year-olds that includes the impact supplement (www.sdqinfo.org). We will also include the SDQ for teachers (useful for children from 4 to 17 years old) in order to cover the whole age group and have a second measure for the validation process. Both versions use the original three-option Likert response format: therefore, the score on each subscale ranged from 0 to 10 . The first four subscales yield a total difficulties score. We hope to receive permission and pay the licence fee to Youthinmind Ltd to create our own online version of the SDQ.

- 'Specific phobia' subscale of Spence Children's Anxiety Scale $(S C A S))^{52}$ We used the specific phobia subscale that consists of five items with four Likert alternatives $(0=$ never, $1=$ sometimes, $2=$ often and $3=$ always $)$ with an average value of internal consistency of. 64 , per a meta-analytic review with 32 studies. ${ }^{53}$

- Children's Revised Impact of Event Scale (CRIES). ${ }^{54}$ The CRIES is a screening scale used to measure PTSD in children aged over 8 years. It consists of eight items with the same four Likert alternatives as above.

\section{Suicidality}

Self-Injurious Thoughts and Behaviours Interview (SITBI). ${ }^{55}$ Suicidality items will be taken mostly from the SITBI. With 24 items in nine modules examining death wishes, suicidal ideation, suicide plans and suicide attempts. Several questions are also asked about non-suicidal self-injury. Suicidality in the past 12 months and over one's lifetime will be assessed.

\section{Mental disorders}

The Anxiety Disorders Interview Schedule for DSM-IV: Child Version $(A D I S-I V-C) .^{56}$ This is a semi-structured interview that assesses current and lifetime child psychopathology per DSM-IV criteria in children aged 6 to 18 years. It is designed to assess anxiety disorders, however it also includes sections for the diagnosis of mood and externalising disorders, and a screening section for substance abuse, schizophrenia, selective mutism, eating and somatoform disorders. It also allows for obtaining relevant information about school refusal, interpersonal relationships, specific areas of interference and psychosocial stressors. For our study, we will use the Spanish version for children by Silverman, Albano and Sandin, ${ }^{57}$ which has good psychometric properties. We will only use components that assess anxiety and mood disorders. Previous studies have indicated adequate reliability for all disorders evaluated $\left(\alpha_{\mathrm{s}}=0.75-.92\right)$.

\section{Subjective well-being}

Mental health and well-being

- DetectaWeb-Well-being Scale. This questionnaire is a reduced web-based screening questionnaire for wellbeing based on the pool of items from other measures of well-being and mental health. It consists of 15 
Table 1 Study variables and assessment timing. The DetectaWeb Project for the detection of the mental health continuum

\begin{tabular}{lllllll}
\hline Section & Subsections/variables & Stage 1 & Stage 2 & Stage 3 & Stage 4 & Original instrument \\
\hline Section 1 & Socio-demographic data & & & & & \\
& Age & $*$ & $*$ & $*$ & $*$ & Ad hoc scale \\
& Sex & $*$ & $*$ & $*$ & $*$ & \\
& Nationality & $*$ & $*$ & $*$ & $*$ & $*$ \\
& Race & $*$ & $*$ & $*$ & $*$ & $*$ \\
& Grade & $*$ & $*$ & $*$ & $*$ & FAS
\end{tabular}

\begin{tabular}{|c|c|c|c|c|c|c|}
\hline \multicolumn{7}{|c|}{ Distress } \\
\hline \multirow[t]{12}{*}{ Section 2} & Internalising symptoms & & & & & \\
\hline & Anxiety symptoms & & & & & \\
\hline & SP & & & & & \\
\hline & SoPh & * & * & * & * & DetectaWeb-Distress \\
\hline & $\mathrm{Pd} / \mathrm{Ag}$ & * & * & * & * & SP subscale SCAS \\
\hline & PTSD & * & * & * & * & CRIES \\
\hline & OCD & * & * & * & * & \\
\hline & Mood & * & * & * & * & \\
\hline & MD & * & * & * & * & \\
\hline & Prosocial behaviour & & & * & * & SDQ \\
\hline & & & & * & * & \\
\hline & & & & * & * & \\
\hline \multirow[t]{5}{*}{ Section 3} & Externalising symptoms & & & & & \\
\hline & Conduct problems & & & & & \\
\hline & Hyperactivity & & & & * & SDQ \\
\hline & & & & & * & \\
\hline & & & & & * & \\
\hline \multirow[t]{8}{*}{ Section 4} & Mental disorders & & & & & \\
\hline & Anxiety disorders & & & & & \\
\hline & SAD & & & * & * & ADIS-IV-C \\
\hline & $\mathrm{SP}$ & & & * & * & \\
\hline & SoPh & & & * & * & \\
\hline & GAD & & & * & * & \\
\hline & $\mathrm{Pd} / \mathrm{Ag}$ & & & * & * & \\
\hline & PTSD & & & * & * & \\
\hline
\end{tabular}




\begin{tabular}{|c|c|c|c|c|c|c|}
\hline Section & Subsections/variables & Stage 1 & Stage 2 & Stage 3 & Stage 4 & Original instrument \\
\hline & OCD & & & * & * & \\
\hline & Mood disorders & & & & & \\
\hline & MD & & & * & * & \\
\hline & $\mathrm{DD}$ & & & * & * & \\
\hline \multicolumn{7}{|c|}{ Well-being } \\
\hline \multirow[t]{5}{*}{ Section 5} & Mental health & * & * & * & * & DetectaWeb-Well-being \\
\hline & & & & & & Scale \\
\hline & & & & & & $\mathrm{MHI}$ \\
\hline & & & & & & MHI-5 \\
\hline & & & & & & WHO-5 \\
\hline Section 6 & Health-related quality of life & * & * & * & * & Kidscreen-10 Index \\
\hline \multirow[t]{8}{*}{ Section 7} & Well-being & * & * & * & * & DetectaWeb-Well-being \\
\hline & & & & & & Scale \\
\hline & & & & & & $\mathrm{MHI}$ \\
\hline & & & & & & MHI-5 \\
\hline & & & & & & WHO-5 \\
\hline & & & & & & SCWBS \\
\hline & & & & & & SWEMWBS \\
\hline & & & & & & MHC-SF \\
\hline Section 8 & Social emotional health/covitality & * & * & * & * & SEHS-Secondary and Primary \\
\hline Section 9 & Emotional intelligence & * & * & * & * & TEIQue-CSF \\
\hline Section 10 & Personality & * & * & * & * & TIPI-CA \\
\hline Section 11 & Social integration & & * & * & & $\begin{array}{l}\text { CSN, CSP, \& CSA } \\
\text { Bull-S }\end{array}$ \\
\hline
\end{tabular}

${ }^{\star}$ Factor included in stage.

FAS, The Family Affluence Scale; ADIS-IV-C, The Anxiety Disorders Interview Schedule for DSM-IV: Child Version; Bull-S, Bulls questionnaire; CRIES, Children's Revised Impact of Event Scale; CSN, CSP and CSA, Sociometric Questionnaire for Children, Pre-adolescents, and Adolescents; DD, persistent depressive disorder or dysthymic disorder; GAD, generalised anxiety disorder; MD, major depression; MHCSF, Mental Health Continuum-Short Form; SEHS-Secondary and MHI-5, Mental Health Inventory (5five items); MHI, Mental Health Inventory (38 items); OCD, obsessive compulsive disorder; Pd/Ag, panic disorder/agoraphobia; Primary, Social Emotional Health Surveysecondary and primary; PTSD, posttraumatic stress disorder; RCADS-30, Revised Child Anxiety and Depression Scale, 30-item version; SAD, separation anxiety disorder; SCWBS, Stirling Children's Well-being Scale; SDQ, The Strengths and Difficulties Questionnaire; SES, The Hollingshead scale; SITBI, Self Injurious Thoughts and BehaviorsBehaviours Interview; SoPh, social phobia; SP subscale SCAS, specific phobia subscale of Spence Children's Anxiety Scale; SP, specific phobia; SWEMWBS, Short version of Warwick-Edinburgh Mental Well-Being Scale; TEIQue-CSF, TraitEmotional Intelligence Questionnaire-Child Short Form; TIPI-CA, Spanish adaptation of the Ten-item Personality Inventory; WHO-5, 5-item World Health OrganizationWHO Well-Being Index.

items that assess well-being. It follows a Likert-type response format $(0=$ never, $1=$ sometimes, $2=$ often and $3=$ always). This questionnaire is in the process of validation, however preliminary data indicates a Cronbach's alpha of .89 .

- Mental Health Inventory (MHI) and MHI- $5 .^{5859}$ The MHI is a 38 -item measure of psychological distress and wellbeing, developed for use in adult general populations. Its factor structure for adults is psychological distress (anxiety, depression and loss of behavioural emotional control) and psychological well-being, with general positive affect and emotional ties as subscales. In our study, the aim is to adapt it for use with children and adolescents. Our own preliminary data indicate that its factor structure consists of two factors: distress (23 items) and well-being (15 items).

The MHI $-5^{59}$ is a short version of the MHI (38 items). The MHI-5 was also developed for use in the general population, including items assessing psychological well-being. This inventory consists of five items about mood over the past month, measuring the presence of psychological well-being (two items) and the absence of psychological distress (three reversed items). Both the MHI and MHI-5 use a six-point response system. In our adaptation study, the response format will be adapted to a four-point Likert-type format $(0=$ never, 1 =sometimes, $2=$ often and $3=$ always). In both versions, a higher score indicates better mental health. 
- MHC-Short Form (MHC-SF).$^{60}$ The MHC-SF consists of 14 items that were chosen as the most prototypical items representing the construct definition for each facet of well-being. Three items were chosen (happy, interested in life and satisfied) to represent emotional well-being, six items (one item from each of the six dimensions) were chosen to represent psychological well-being and five items (one item from each of the five dimensions) were chosen to represent social wellbeing. The response option for the short form was changed to measure the frequency that respondents experienced each symptom of positive mental health, and thereby provided a clear standard for the assessment and a categorisation of levels of positive mental health. This instrument has received psychometric support for its use with adolescents. ${ }^{61} 62$ In this study, we will adapt the MHC-SF for its use with children.

Health-Related Quality of Life

Kidscreen-10 Index. ${ }^{63}$ This is a 10 -item questionnaire that assesses subjective Health-Related Quality of Life (HRQL) and well-being for children and adolescents aged 8 to 18 years. For each item, five answer categories ranging from 'never' to 'always' or from 'not at all' to 'extremely' are provided. The index addresses affective symptoms of depressed mood, cognitive symptoms of disturbed concentration, psycho-vegetative aspects of vitality, energy and feeling well, and psychosocial aspects correlated with mental health, such as the ability to experience fun with friends or getting along well at school.

\section{Well-being}

- WHO-5. ${ }^{64}$ It is a short and generic global rating scale measuring subjective well-being. The five items devising the WHO-5 assess aspects such as positive mood, calm/relaxation, activity/vigorousness and general interest, among others. The original questionnaire is measured using the scale: 5 (all the time) to 0 (none of the time). However, for children and adolescents, we will use a simpler rating scale: 3 (always) to 0 (never). A higher score indicates greater well-being.

- Short version of Warwick-Edinburgh Mental Well-Being Scale (SWEMWBS). ${ }^{65}$ This seven-item scale is one of the most used measures of well-being, and it has been validated for the Spanish general population aged under 14 years. ${ }^{66}$

- Stirling Children's Well-being Scale (SCWBS). ${ }^{40} 67$ This 15-item scale was developed by the Stirling Council Educational Psychology Service (UK) as a holistic, positively worded measure of emotional and psychological well-being in children aged 8 to 15 years.

\section{Social emotional health/covitality}

- Social Emotional Health Survey-Secondary (SEHS-Secondary) ${ }^{12}$ and SEHS-Primary. On the one hand, the SEHS is a modification and extension of the Resilience Youth Development Module that is part of the suite of assessments associated with the California Healthy
Kids Survey ${ }^{68}$ The SEHS-S assesses core psychosocial assets based on a higher-order model of 'covitality' that consists of four latent traits (each comprised of three measured subscales, three items per subscale): belief-in-self (with subscales of self-efficacy, self-awareness and persistence); belief-in-others (with subscales of school support, peer support and family coherence); emotional competence (with subscales of emotional regulation, behavioural self-control and empathy); and engaged living (with subscales of gratitude, zest and optimism). ${ }^{12}$ This 36 -item instrument is used with adolescents aged 14 to 18 years. For 10 of the 12 subscales, the students' self-reports are completed using a four-point scale $(1=$ not at all true of me, $2=$ alittle true of me, $3=$ pretty much true of me and $4=$ very much true of me). The gratitude and zest measured subscales use a five-point response scale: ( $1=$ not at all, $2=$ verylittle, $3=$ =somewhat, $4=$ quite a lot, $5=$ extremely). Six previous studies have examined the psychometric properties of the SEHS-S, providing construct validity support for the SEHS-S as a higher-order measurement model. ${ }^{12}$

- SEHS-Primary. ${ }^{68}$ This is a 20 -item scale available for children aged 8 to 13 years and self-rated on a fourpoint scale: $1=$ never, $2=$ sometimes, $3=$ often and $4=$ very often. It assesses core psychosocial strengths based on a second-order model of 'covitality' that consists of four lower-order positive psychology traits measured by four subscales: gratitude, optimism, zest and persistence. In addition, the SEHS-P includes another subscale that measures students' prosocial behaviour at school, which is not part of the covitality construct. This instrument has been validated in elementary school students from the USA. ${ }^{69}$ and is currently being validated in China.

\section{Emotional intelligence}

Trait Emotional Intelligence Questionnaire-Child Short Form (TEIQue-CSF). ${ }^{70}$ This questionnaire is designed to measure global trait emotional intelligence (trait EI) for children aged 8 to 12 years and includes 36 items responded to on a five-point Likert scale ( $1=$ strongly disagree to $5=$ strongly agree). The total score indicates global trait EI. This questionnaire is available, free of charge for academic research purposes, from the London Psychometric Laboratory. We will use the Spanish adaptation by Benito and González-Perez, which is in the process of adaptation by them and our research team.

\section{Personality}

Spanish adaptation of the Ten-Item Personality Inventory (TIPI) ${ }^{71}{ }^{72}$ for its use with children and adolescents (TIPICA) ${ }^{73}$ Personality traits will be measured with this adapted measure. This tool, based on other well-established instruments measuring the big five personality traits, consists of 10 items, each comprising a pair of descriptors that are scored from 1 (strongly disagree) to 7 (strongly agree). Each dimension of personality (ie, extraversion, 
agreeableness, conscientiousness, emotional stability and openness to experience) is represented by two items. The test has been validated for young and middle-age adult populations. This study indicates that the psychometric properties of TIPI-CA are adequate. Some examples of changes in the adaptation for adolescents were the replacement of terms such as 'serena' (calm) with 'tranquila' (quiet), 'colérica' (critical) with 'que critica a los demas' (critical with others), or 'polifacética' (complex) with 'con intereses diversos' (with diverse interests).

\section{Social integration}

Social integration will be assessed by a combination of two sociometric questionnaires:

- Sociometric Questionnaire for Children, Pre-adolescents, and Adolescents (Cuestionario Sociométrico para Niños, Preadolescentes y Adolescentes; CSN, CSP and CSA).${ }^{74} \mathrm{It}$ is based on a nominations method: students are requested to report the three boys or girls of their group who they are more likely to play with, asking them the reasons for their elections and rejections. They provide a rate of rejections and elections: between the number of possible rejections/elections and the total number of students. Per the two-dimensional classification developed by Coie, ${ }^{75}$ one can distinguish five sociometric status: popular, rejected, isolated, controversial and normal.

- Bull-S questionnaire ${ }^{76}$ for measuring the social-affective structure and peer relationships and bullying. The Bull-S test ( ${ }^{76}$ revised in 2002) was designed to measure three general aspects of bullying: sociometric position; bullying characteristics; and situational properties. It is based on peer nominations and self-evaluation. It consists of 15 items grouped around three topics: sociometric position, aggression-victimisation and situational properties.

\section{Adaptation of the questionnaires into Spanish}

Many instruments used in this study already have a Spanish version available and have been used in several previous studies. Examples include the RCADS-30, ${ }^{49}$ the $\mathrm{SDQ}^{77}$ and the SITBI. ${ }^{78}$ Nevertheless, for the adaptations of the remaining instruments and items into Spanish, we have translated and adapted the original scales into Spanish in stage 1. For this, we followed international guidelines and technical standards. ${ }^{79}$ Specifically, we conducted the following steps: the Spanish translations were authorised by the copyright owners; a translation/ back-translation procedure was conducted; (expert judgement to support the content validity (linguistic equivalence and understanding of the items) was developed by researchers specialised in different fields related with our purpose (child and adolescent clinical psychology and psychiatry, psychological assessment, psychometrics, and so on); experts evaluated the correspondence of each item to the conforming dimension/factor to support the content validity of the construct; and the Spanish version of the measures were then administered to a pilot sample of children in stage 2, balanced in sex distribution, to ensure that the item content could be understood by young children. For this, we chose the cognitive interview technique, a qualitative methodology.

\section{Study quality control procedures}

Data quality control procedures will be implemented and the results will be reported on a twice-a-month basis regarding the following aspects: survey participation, duration, and individual- and item-level quality data.

\section{Survey participation}

A table will be included with the number of children registered to the survey and a summary of their participation and completion rate. The table will include a summary of the validation status of registered individuals as provided by each phase. For each phase, we will validate new registrations and will report the causes of exclusion. For each participant, there will be three possible validation statuses: valid (ie, inclusion criteria are fulfilled), excluded (ie, inclusion criteria are not fulfilled) or pending validation.

\section{Survey duration}

A table will report descriptive statistics of the duration time for each school centre and the overall sample. Additionally, the number of individuals below and over a pre-specified threshold will be reported to identify possible outliers. We have set values to flag attention about response quality: a time of less than $15 \mathrm{~min}$ and a time of more than $45 \mathrm{~min}$ (as they correspond to the 25th and 75 th percentiles of the response distribution).

\section{Quality checks at the individual and item levels}

All items from our survey are compulsory, therefore it is not possible for empty responses or the identification of problematic items or skipping errors in the interview. Nevertheless, our assistance at classrooms will reduce the presence of problems in the fulfilment of the survey.

\section{Participant alerts}

Although inquiring about mental disorder such as depression, anxiety or suicidal behaviour does not increase the risk of suicidal behaviour, it is important to minimise any possible risk. ${ }^{80}$ Therefore, at the end of the survey administration, our platform will display a summary that provides information about the mental health risk of anonymous surveyed participants. We will follow the Spanish current legislation acting properly in situations of risk for the minor, so that a specific alert will warn us to intervene at risk situations for minors.

\section{Clinical reappraisal}

The instruments used in the DetectaWeb Project for assessing mental disorders symptoms are the RCADS, the SCAS's specific phobia subscale, the CRIES, the SDQ, and so on. However, given that the instrument includes other scales in the process of validation, such as the DetectaWeb-Distress Scale, as well as the fact that they 


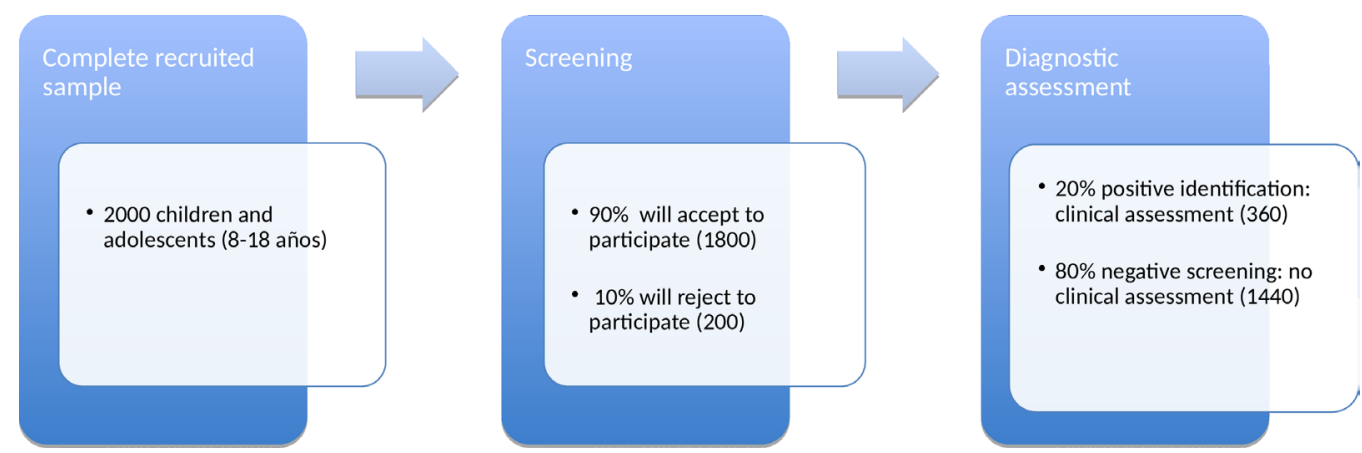

have not been tested among Spanish children and adolescents, we decided to perform a clinical reappraisal.

Therefore, the adapted Spanish version of the Anxiety Disorders Interview Schedule for DSM-IV: Child Version (ADIS$I V-C)^{57}$ will be administered to a subsample of respondents shortly after the completion of the online survey to assess diagnostic concordance. Administration of the ADIS-IV-C will be completed by trained mental health professionals face-to-face. The ADIS-IV-C is a semi-structured clinical diagnostic interview and is considered the gold standard for clinical diagnoses of anxiety and depression disorders among children and adolescents. ${ }^{57}$ Interviewers will be blinded to participants' mental health status. Eligible respondents will be selected after completion of online surveys in stages 3 and 4 . For this study, a random sample totalling $10 \%$ of the child interviews will be audiotaped and scored by another interviewer who will be masked as to adolescents' screening scores. Interviews will be conducted by clinical psychology graduate students who will be trained in a 12-hours workshop and supervised by a licensed clinical psychologist.

\section{Plan to recruit school and clinical samples}

\section{School-based samples}

To ensure sample representativeness, random cluster sampling will be used (figure 2).

One of the most important prevalence estimates in the context of the community child population, including more than 700 families, suggested that about $20 \%$ of children ( 8 to 17 years) were above the clinical cut-off in a screening measure of anxiety. ${ }^{81}$ Piqueras et $\mathrm{al}^{82}$ found that $9.17 \%$ of adolescents exceeded cut-off points for SAD after examining over 10000 adolescents for five years. Cuijpers et $\mathrm{al}^{37}$ found a prevalence of $20 \%-23 \%$ for depressive disorders using an online screening programme for adolescents. Kuo et $\mathrm{al}^{83}$ indicated that $5 \%-20 \%$ of the school-based sample from its screening programme for evolutionary trajectories exceeded cut-offs for depressive symptoms or behaviour problems. Therefore, we expect to detect $20 \%$ (conservative estimate) of participants with high levels of psychological distress (anxiety and/ or depression) and/or low levels of well-being, therefore approximately 400 children would move to the next phase. Given the average rates of refusal to participate in screening interventions and the estimated positive detection, we developed a flow chart on a hypothetical student sample (see flowchart below) informed by the above studies. Nevertheless, it ensures clinical and statistical significance, and the feasibility of the project.

\section{Clinical sample}

The sample will consist of approximately 150 users aged 8 to 18 years, of both sexes, recruited from child mental health units of the Spanish National Public Health System. To test the diagnostic accuracy of the project, the first 100 children and adolescents who attend CAMHU reporting complaints of anxiety and/or depression will be enrolled, while the following 100 children and adolescents with other different complaints (conduct problems, attention deficit hyperactivity disorder, and so on) will be included to test the ability to discriminate between different types of mental disorders (figure 3).

\section{Analysis plan}

\section{Survey response and participation bias assessment}

We expect a participation rate be around $90 \%$ according to rates from our own previous studies, however this will depend on parental consent. Study representativeness will depend on a high level of participation and that respondents will be representative of the target population (concerning sex, age, geographic area and school type). We expect the response rate to the survey to be near $100 \%$, since all answers will be mandatory, and the system will notify the participant if an item is not answered. We will also have some items and questionnaires conditioned per age and response type.

\section{Validation of the DetectaWeb Project}

The objective of our study is the creation and validation of a programme that prevents, detects and promotes the MHC. Therefore, we have divided our study into four phases, which will require different data analysis. In the first phase, the inter-rater agreement, such as the Aikens' $\mathrm{V}$ and qualitative analyses related to item comprehensibility, will be measured for all instruments. Consequently, we will select items that measure the MHC effectively. 

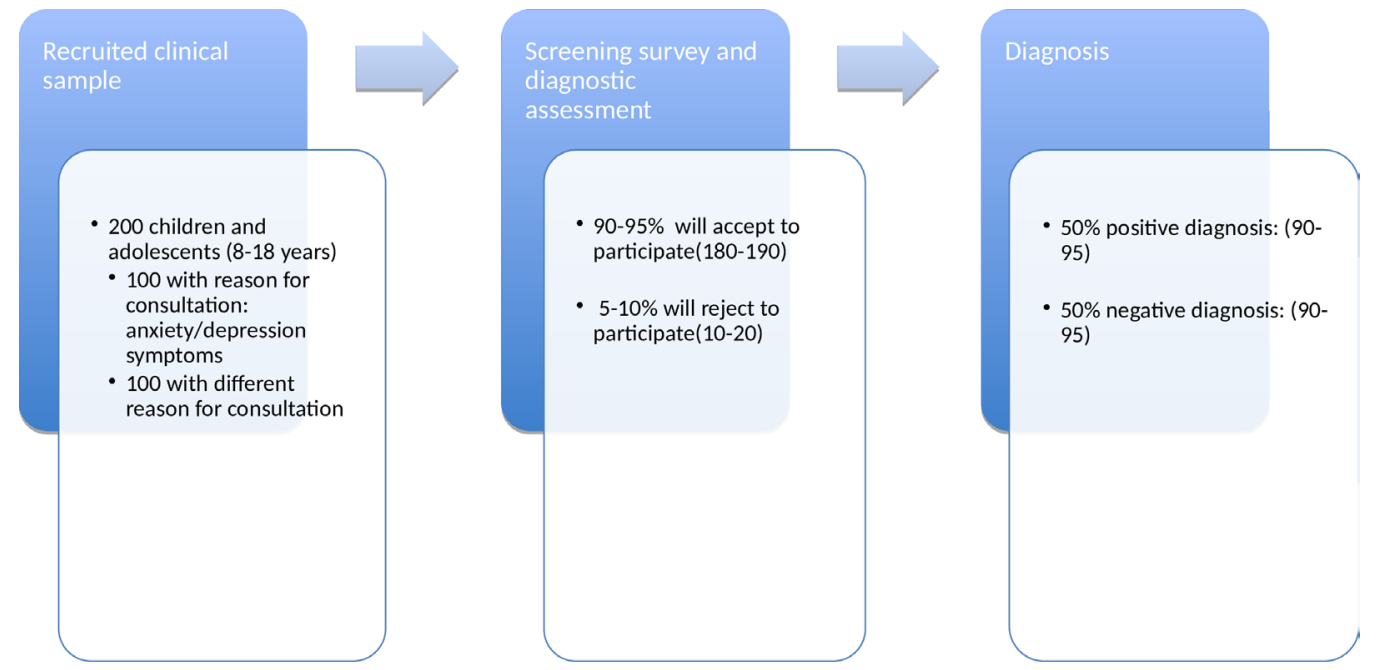

Figure 3 Flowchart of the screening programme phases for the clinical sample (estimates based on previous studies).

In the second phase, we will use a pilot study to test our assessment protocol by conducting a descriptive analysis of the items, an exploratory factor analysis, estimations of internal consistency, an analysis of evidence of validity criteria through correlations with other measures and calculate the differences in the scores per sex and age. In the third phase, we will conduct different data analysis such as confirmatory factor analysis (CFA) to examine factor structure and multi-group CFA to contrast factor invariance across sex and age; estimates of internal consistency; analysis of validity criteria by examining correlations between measurements (convergent validity); calculating logistic regressions (odds ratios) and using contingency tables and ROC curves as well as sensitivity/specificity values by means of clinical diagnoses with ADIS-IV-C; and comparisons of scores based on sex and age. During stage 3 , we will also analyse the prevalence of DetectaWeb-Distress: anxiety, depression and suicidality and DetectaWebWell-being: and psychological, emotional and social well-being. Prevalence rates and MHC modelling will be calculated here. Finally, in the fourth phase, we will conduct the same analyses as in the third phase, but with a clinical population. In the second to the fouth phases, participants' cut-off scores will be calculated. Finally, once all phases have ended, we will conduct a study of analysis of the cost-effectiveness of our online protocol.

\section{STRENGTHS AND LIMITATIONS OF THIS STUDY}

The DetectaWeb Project will provide a protocol allowing to prevent, detect and promote MHC in children and adolescents, providing the ability to detect and intervene prematurely and/or prevent future emotional disorders, as well as to promote well-being.

- The use of an online assessment protocol presents several advantages, such as it will allow a reduction in costs; increase the access, rapid assessment an analysis of almost immediate results; and offer an appealing tool to the target populations.
- These advantages will enable greater ease in two ways: it will be useful for schools as a tool for identifying children with lower levels of mental health as well as for parents and educators who will receive the necessary information so that their children can receive the necessary care and/or enhance their welfare; and its inclusion at CAMHU will be useful as a tool for assessment or screening, to determine the symptoms and severity, to decide which professional has to be referred to for treatment, to select therapeutic targets and to assess therapeutic change in a more complete way than what was traditionally focused on.

- In this study, a limitation is the representativeness of the sample, since it will take place in a single province of Spain. Therefore, its external validity will be limited, as they still are part of a convenience rather than random sampling strategy. However, there is geographical variation in the province, and the incidence and predictive models will not be affected by this bias. Furthermore, there is a plan to later extend this project to other regions of Spain.

\section{ETHICS AND DISSEMINATION SECTION \\ Confidentiality and ethical issues}

We will receive assistance by a firm of lawyers specialising in data protection and information security to ensure compliance with current regulations in Spain: General Data Protection Regulation, article number 5 of Law 15/1999 of 13 December, adopted by Royal Decree 1720/2007 of 21 December; Law 41 / 2002 of 14 November on the autonomy of patients and rights and obligations with regard to clinical information and documentation; and Spanish Minor Law, according to which we should act properly in situations of risk for the minor (Art. 7 . Interventions at risk situations for Minors).

Furthermore, the DetectaWeb Project has been approved by the Ethical Committee for research projects (Órgano Evaluador de Proyectos, OEP) from the Vicerrectorado for Research and Technological Development 
from the Miguel Hernandez University (reference numbers DPS-JPR-001-10 and DPS.JPR.02.14). In addition, we have obtained approvals by the local Ethical Committees for Clinical Research from the University General Hospital of San Juan of Alicante, the University General Hospital of Elche and the University General Hospital of Elda (Alicante). This study follows the code of ethics and the international ethical guidelines for biomedical research involving human subjects per the Declaration of Helsinki of the World Medical Association (Seoul 2008 revision) and standards of good clinical practice of the European Union.

Finally, an online informative note is provided for each respondent at the beginning of the survey. This note indicates that information collected will be treated as strictly confidential and private, and that their data will be collected through a database hosted in a server property of the Department of Health Psychology of Miguel Hernandez University.

\section{Potential impact}

The DetectaWeb Project has the potential to provide a valid, innovative and useful web-based assessment project for the MHC of children and adolescents. The project will emphasise the development and validation of specific MHC measures, and provide useful knowledge concerning the prevalence rates of mental health and associations with different variables.

Compared with measures and early detection programmes for psychopathology or mental disorders, there are very few studies examining the usefulness of the MHC through a wide approach to assess both mental health and emotional well-being in children and adolescents. Consequently, there is a need for the development of a comprehensive assessment of mental health in schools. This project is intended to lead to the design of an evidence-based protocol of assessment to evaluate complete mental health (both mental health disorders and mental well-being).

In general, it represents the first study about the MHC (distress and well-being) in Spanish children and adolescents. In addition, the DetectaWeb Project will provide useful data for the development of prevention programmes for adolescents' mental health, for improving early detection for high-risk children and adolescents at school, and promoting the well-being and psychological skills that will enhance mental health.

Finally, it should be noted that this project could be the first step to provide evidence for an assessment protocol focused on MHC in children and adolescents, which would support the subsequent development of preventative interventions. These interventions could be based on social emotional learning, positive psychology literature, childhood resilience literature or emotional intelligence interventions for the promotion and building of resilience and strengths among children and adolescents at risk for mental health problems.
Results of the study will be communicated during national and international meetings in the field of clinical and health psychology with children and adolescents. Publications will be sought in journals usually read by psychologists or psychiatrists involved in the development of epidemiological studies and interventions for mental health promotion based on resilience building for children and adolescents.

Contributors All authors have contributed to the design and development of the protocol. The concept of the protocol was formulated by JAP and PC. The acquirement of the funding and permissions for the research was provided by JAP. The drafting of the manuscript was done by JAP. MRR, MGO, TRJ and AEMG are collecting the data, will perform the statistical analysis and assisted in drafting of the manuscript. Critical revision of the protocol was performed by MRR, MGO, TRJ, AEMG AND PC.

Funding Detecta Web Project is a funded project by the Vicerrectorado for Research and Technological Development from Miguel Hernández University (BANCAJA-UMH, call 2010), the Department of Health Care (Consejeria de Sanidad) from the Valencian Community (SMI 10/2014), and and a grant for thehiring of predoctoral research assistant granted the third author (ProgramVALi+d, Department of Education, Research, Culture and Sport from the ValencianCommunity).

Competing interests None declared.

Ethics approval Ethical Committee for research projects (Órgano Evaluador de Proyectos, 0EP) from the Vicerrectorado for Research and Technological Development from the Miguel Hernandez University (reference numbers DPS-JPR001-10 and DPS.JPR.02.14).

Provenance and peer review Not commissioned; externally peer reviewed.

Open Access This is an Open Access article distributed in accordance with the Creative Commons Attribution Non Commercial (CC BY-NC 4.0) license, which permits others to distribute, remix, adapt, build upon this work non-commercially, and license their derivative works on different terms, provided the original work is properly cited and the use is non-commercial. See: http://creativecommons.org/ licenses/by-nc/4.0/

(C) Article author(s) (or their employer(s) unless otherwise stated in the text of the article) 2017. All rights reserved. No commercial use is permitted unless otherwise expressly granted.

\section{REFERENCES}

1. World Health Organization. Mental health: a state of well-being 2013 Report of the WHO Department of Mental Health. 2013 http://www. who.int/features/factfiles/mental_health/en/ (accessed 20 Mar 2017).

2. World Health Organization. Mental health: strengthening our response. Fact sheet 220. 2016 http://www.who.int/mediacentre/ factsheets/fs220/en/ (accessed 20 Mar 2017).

3. Keyes CL. The mental health continuum: from languishing to flourishing in life. J Health Soc Behav 2002;43:207-22.

4. Keyes CLM. Mental illness and/or mental health? Investigating axioms of the complete state model of health. J Consult Clin Psychol 2005;73:539-48.

5. Keyes CLM. Promoting and protecting mental health as flourishing: a complementary strategy for improving national mental health. Am Psychol 2007;62:95-108.

6. Ryff CD, Keyes CLM. The structure of psychological well-being revisited. J Pers Soc Psychol 1995;69:719-27.

7. Keyes CLM. Mental health in adolescence: is America's youth flourishing? Am J Orthopsychiatry. Educational Publishing Foundation 2006;76:395.

8. Eryılmaz A. A model of subjective well-being for adolescents in high school. J Happiness Stud 2012;13:275-89.

9. Kelly RM, Hills KJ, Huebner ES, et al. The longitudinal stability and dynamics of group membership in the dual-factor model of mental health: psychosocial predictors of mental health. Can J Sch Psychol 2012;27:1-19.

10. Suldo SM, Shaffer EJ. Looking beyond psychopathology: the dual-factor model of mental health in youth. School Psych Rev 2008;37:52-68. 
11. Suldo S, Thalji A, Ferron J. Longitudinal academic outcomes predicted by early adolescents' subjective well-being, psychopathology, and mental health status yielded from a dual factor model. J Posit Psychol 2011;6:17-30.

12. Furlong MJ, You S, Renshaw TL, et al. Preliminary development and validation of the social and emotional health survey for secondary school students. Soc Indic Res 2014;117:1011-32.

13. Furukawa TA, Kessler RC, Slade T, et al. The performance of the K6 and K10 screening scales for psychological distress in the Australian National Survey of Mental Health and Well-Being. Psychol Med 2003;33:357-62.

14. Kessler RC, Andrews G, Colpe LJ, et al. Short screening scales to monitor population prevalences and trends in non-specific psychological distress. Psychol Med 2002;32:959-76.

15. van Ballegooijen W, Riper $\mathrm{H}$, Cuijpers $\mathrm{P}$, et al. Validation of online psychometric instruments for common mental health disorders: a systematic review. BMC Psychiatry 2016;16:45.

16. Clark LA, Watson D. Distress and fear disorders: An alternative empirically based taxonomy of the "mood" and "anxiety" disorders. Br J Psychiatry 2006;189:481-3.

17. Krueger RF. The structure of common mental disorders. Arch Gen Psychiatry 1999;56:921-6.

18. Watson D. Rethinking the mood and anxiety disorders: a quantitative hierarchical model for DSM-V. J Abnorm Psychol 2005;114:522-36.

19. Polanczyk GV, Salum GA, Sugaya LS, et al. Annual research review: A meta-analysis of the worldwide prevalence of mental disorders in children and adolescents. J Child Psychol Psychiatry 2015;56:345-65.

20. Nock MK, Hwang I, Sampson NA, et al. Mental disorders, comorbidity and suicidal behavior: Results from the National Comorbidity Survey Replication. Mol Psychiatry 2010;15:868-76.

21. Cougle JR, Keough ME, Riccardi CJ, et al. Anxiety disorders and suicidality in the National Comorbidity Survey-Replication. $J$ Psychiatr Res 2009;43:825-9.

22. Ten Leading Causes of Death by Age Group, United States. Report of the Centers for Disease Control and Prevention, and US Department of Health and Human Services. 2014 (accessed 20 Mar 2017).

23. Vázquez C, Hervás G, Rahona JJ, et al. Bienestar psicológico y salud: Aportaciones desde la Psicología Positiva. Anu Psicol Clínica y la Salud 2009;5:15-28.

24. Ryff CD. Happiness is everything or is it?J Personal Social Psychol 1989;57:1069-81.

25. Fellow R, Stewart-Brown S, Taggart F. Warwick-Edinburgh Mental Well-being Scale (WEMWBS). User Guid Version 2. 2015.

26. Diener E. Subjective well-being: The science of happiness and a proposal for a national index. Am Psychol 2000;55:34-43.

27. Diener E, Lucas RE, Oishi S. Subjective well-being. Handb Posit Psychol. 1984;95:63-73.

28. Keyes CLM. The nature and importance of positive mental health in America's adolescents. Handb Posit Psychol Sch 2009:9-23.

29. Neil AL, Christensen H. Efficacy and effectiveness of school-based prevention and early intervention programs for anxiety. Clin Psychol Rev 2009;29:208-15.

30. Andersson G, Carlbring P, Kaldo V, et al. Screening of psychiatric disorders via the Internet. A pilot study with tinnitus patients. Nord $\mathrm{J}$ Psychiatry 2004;58:287-91.

31. Cuijpers P, Boluijt $P$, van Straten A. Screening of depression in adolescents through the Internet : sensitivity and specificity of two screening questionnaires. Eur Child Adolesc Psychiatry 2008;17:32-8.

32. Donker T, Straten Avan, Marks I, et al. A brief web-based screening questionnaire for common mental disorders: development and validation. J Med Internet Res 2009;11:e19.

33. Hoffmann M, Rojas G, Martínez V. Prevención, detección, tratamiento o seguimiento en salud mental de adolescentes a través del uso de Internet: una revisión sistemática cualitativa. Rev Med Chil 2014;142:494-500.

34. Mogle J. Internet-based assessment. The Encyclopedia of adulthood and aging, 2015:1-5.

35. Fox JK, Halpern LF, Forsyth JP. Mental health checkups for children and adolescents: A means to Identify, prevent, and minimize suffering associated with anxiety and mood disorders. Clin Psychol Sci Pract 2008;15:182-211

36. Cuijpers $P$, Boluijt $P$, van Straten A. Screening of depression in adolescents through the Internet : sensitivity and specificity of two screening questionnaires. Eur Child Adolesc Psychiatry 2008;17:32-8.

37. Muñiz J, Elosua P, Hambleton RK. Directrices para la traducción y adaptación de los tests: Segunda edición. Psicothema 2013:25:151-7.
38. The International Test Commission. International guidelines on computer-based and internet-delivered testing. Int J Testing 2006;6:143-71.

39. Leong FTL, Bartram D, lliescu D. The ITC International Handbook of Testing and Assessment. Oxford University Press 2016.

40. Carter G, Liddle I. Emotional and psychological wellbeing in children The standardisation of the Stirling children's wellbeing scale. Stirling Council. 2011. Retrieved from http://www. friendsforlifescotland. org/ site/SCWBS\% 20Report. pdf.

41. Furlong MJ, You S, Renshaw TL, et al. Preliminary development and validation of the social and emotional health survey for secondary school students. Soc Indic Res 2014:117:1011-32.

42. Cooper A, Petrides KV. A psychometric analysis of the Trait Emotional Intelligence Questionnaire-Short Form (TEIQue-SF) using item response theory. J Pers Assess 2010;92:449-57.

43. Oros LB. Nuevo cuestionario de emociones positivas para niños. An Psicol 2014;30:522-9.

44. Deighton J, Croudace T, Fonagy P, et al. Measuring mental health and wellbeing outcomes for children and adolescents to inform practice and policy: a review of child self-report measures. Child Adol Psych Men 2014;8:14.

45. Currie CE, Elton RA, Todd J, et al. Indicators of socioeconomic status for adolescents: the WHO Health Behaviour in School-aged Children Survey. Health Educ Res 1997;12:385-97.

46. Boyce W, Torsheim T, Currie C, et al. The family affluence scale as a measure of national wealth: validation of an adolescent self-report measure. Soc Indic Res 2006;78:473-87.

47. Hollingshead AB. Four factor index of social status (Unpublished Working Paper, 1975). Yale J Sociol 2011;8:21-52.

48. García-Olcina M, Piqueras J, Martínez-González A. Datos preliminares de la validación del Cuestionario de Detección vía Web para los trastornos emocionales (DETECTA-WEB) en adolescentes españoles. Rev Psicol Clínica con Niños y Adolesc 2014;1:69-77.

49. Chorpita BF, Yim L, Moffitt C, et al. Assessment of symptoms of DSM-IV anxiety and depression in children: a revised child anxiety and depression scale. Behav Res Ther 2000;38:835-55.

50. Sandín B, Chorot P, Valiente RM, et al. Development of a 30-item version of the revised child anxiety and depression scale. Rev Psicopatología y Psicol Clínica 2010;15:165-78.

51. Goodman R. The Strengths and Difficulties Questionnaire: a research note. J Child Psychol Psychiatry 1997;38:581-6.

52. Orgilés M, Méndez X, Spence SH, et al. Spanish validation of the Spence Children's Anxiety Scale. Child Psychiatry Hum Dev 2012;43:271-81.

53. Orgilés M, Fernández-Martínez I, Guillén-Riquelme A, et al. A systematic review of the factor structure and reliability of the Spence Children's Anxiety Scale. J Affect Disord 2016;15:333-40.

54. Perrin S, Meiser-Stedman R, Smith P. The Children's Revised Impact of Event Scale (CRIES): Validity as a screening instrument for PTSD. Behav Cogn Psychoth 2005;33:487-98.

55. Nock MK, Holmberg EB, Photos VI, et al. Self-injurious thoughts and behaviors Interview: development, reliability, and validity in an adolescent sample. Psychol Assess 2007;19:309-17.

56. Silverman WK, Albano AM, Sandín B. ADIS-IV: C: entrevista para el diagnóstico de los trastornos de ansiedad en niños según el DSMIV: entrevista para el niño=(anxiety disorders interview schedule for DSM-IV: child version: child interview shedule). Madrid: Klinik, 2003.

57. Silverman WK, Albano AM, Sandín B. Entrevista clínica ADIS-IV-C: entrevista para el niño. Madrid: UNED-Klinik, 2001.

58. Veit CT, Ware JE. The structure of psychological distress and wellbeing in general populations. J Consult Clin Psychol 1983;51:730-42.

59. Berwick DM, Murphy JM, Goldman PA, et al. Performance of a fiveitem mental health screening test. Med Care 1991;29:169-76.

60. Keyes CLM, Wissing M, Potgieter JP, et al. Evaluation of the mental health continuum-short form (MHC-SF) in Setswana-speaking South Africans. Clin Psychol Psychother 2008;15:181-92.

61. Guo C, Tomson G, Guo J, et al. Psychometric evaluation of the Mental Health Continuum-Short Form (MHC-SF) in Chinese adolescents - a methodological study. Health Qual Life Outcomes 2015;13:198.

62. Lim Y-J. Psychometric characteristics of the Korean Mental Health Continuum-Short Form in an adolescent sample. J Psychoeduc Assess 2014;32:356-64.

63. Ravens-Sieberer U. European KIDSCREEN Group. The Kidscreen questionnaires: quality of life questionnaires for children and adolescents; handbook. Pabst Science Publ 2006.

64. World Health Organization (WHO). Well-being measures in primary health care: the DepCare project. Copenhagen: Denmark WHO Reg Off Eur, 1998:b32.

65. Stewart-Brown S, Platt S, Tennant A, et al. The Warwick-Edinburgh Mental Well-being Scale (WEMWBS): a valid and reliable tool for 
measuring mental well-being in diverse populations and projects. $J$ Epidemiol Community Health 2011;65:A38-A39.

66. Castellví P, Forero CG, Codony M, et al. The Spanish version of the Warwick-Edinburgh Mental Well-Being Scale (WEMWBS) is valid for use in the general population. Qual Life Res 2014;23:857-68.

67. Liddle I, Carter GFA. Emotional and psychological well-being in children: the development and validation of the Stirling Children's Well-being Scale. Educ Psychol Pract 2015;31:174-85.

68. Furlong MJ, Ritchey KM, O'Brennan LM. Developing norms for the California Resilience Youth Development Module: Internal assets and school resources subscales. Calif Sch Psychol 2009;14:35-46.

69. Furlong MJ, You S, Renshaw TL, et al. Preliminary development of the Positive Experiences at School Scale for Elementary School Children. Child Indic Res 2013;6:753-75.

70 Mavroveli S, Petrides KV, Shove C, et al. Investigation of the construct of trait emotional intelligence in children. Eur Child Adolesc. Psychiatry Springer 2008;17:516-26.

71. Renau V, Oberst U, Gosling SD, et al. Translation and validation of the ten-item-personality inventory into Spanish and Catalan. Rev Psicol Ciències l'Educació i l'Esport 2013;3:85-97.

72. Romero E, Villar P, Gómez-Fraguela JA, et al. Measuring personality traits with ultra-short scales: A study of the Ten-Item Personality Inventory (TIPI) in a Spanish sample. Pers Individ Dif 2012;53:289-93.

73. Piqueras JA, Rivera -Riquelme M, García -Oliva C, et al. Spanish adaptation of the Ten-Item Personality Inventory (TIPI) for its use with children and adolescents (TIPI-CA). 2017. Manuscript submitted.

74. Diaz-Aguado M. Children with emotional difficulties. Evaluation instruments [Niños con dificultades socioemocionales. Instrumentos de evaluación].Madrid: Ministerio de Asuntos Sociales, 1995.
75. Coie JD, Dodge KA, Coppotelli H. Dimensions and types of social status: A cross-age perspective. Dev Psychol 1982;18:557-70.

76. Cerezo F, Bull- S. Albor?Cohs MB, Test de Evaluación de la Agresividad entre Escolares, 2000.

77. García P, Goodman R, Mazaria J, et al. El cuestionario de Capacidades y Dificultades. Rev Psiquiatr Infanto-Juv 2000;1:12-17.

78. García-Nieto R, Blasco-Fontecilla H, Paz Yepes M, et al. Translation and validation of the "Self-injurious thoughts and behaviors Interview" in a Spanish population with suicidal behaviour. Rev Psiquiatr Salud Ment 2013;6:101-8.

79. Hambleton RK, Merenda PF, Spielberger CD. Adapting educational and psychological tests for cross-cultural assessment. Psychology Press 2005

80. Gordon R, Angus K. Review of literature to identify whether encouraging people to talk about suicidal feelings is associated with any known/identified positive or negative outcomes and identify any similar/relevant awareness raising/social marketing campaigns \& their impact. Inst Soc Mark 2007.

81. Chavira DA, Stein MB, Bailey K, et al. Child anxiety in primary care: Prevalent but untreated. Depress Anxiety 2004;20:155-64.

82. Piqueras JA, Olivares J, López-Pina JA. A new proposal for the subtypes of social phobia in a sample of Spanish adolescents. $J$ Anxiety Disord 2008;22:67-77.

83. Kuo E, Vander Stoep A, McCauley E, et al. Cost-effectiveness of a school-based emotional health screening program. J Sch Health 2009;79:277-85. 